\title{
Stability of density-stratified viscous Taylor-Couette flows
}

\author{
D. Shalybkov ${ }^{1,2}$ and G. Rüdiger ${ }^{1,3}$ \\ 1 Astrophysikalisches Institut Potsdam, An der Sternwarte 16, 14482 Potsdam, Germany \\ e-mail: gruediger@aip.de \\ 2 A.F. Ioffe Institute for Physics and Technology, 194021 St. Petersburg, Russia \\ 3 Isaac Newton Institute for Mathematical Sciences, 20 Clarkson Road, Cambridge CB3 0EH, UK
}

Received 7 December 2004 / Accepted 21 April 2005

\begin{abstract}
We consider the stability of density-stratified viscous Taylor-Couette flow using the Boussinesq approximation, but without any use of the short-wave approximation. Flows which are unstable according to the Rayleigh criterion $\left(\hat{\mu}<\hat{\eta}^{2}\right.$, with $\hat{\mu}=\Omega_{\text {out }} / \Omega_{\text {in }}$ and $\left.\hat{\eta}=R_{\text {in }} / R_{\text {out }}\right)$, now develop overstable axisymmetric Taylor vortices. However, for the wide-gap container considered here, we find that nonaxisymmetric modes are preferred. These nonaxisymmetric modes are unstable also beyond the Rayleigh line. For such modes the instability condition seems simply to be $\hat{\mu}<1$, as stressed by Yavneh, McWilliams \& Molemaker (2001). However, if the rotation law is too flat, fulfilling the condition $\hat{\mu}>\hat{\eta}$, we never found unstable modes. The Reynolds numbers grow rapidly to very large values as this limit is approached (see Figs. 3 and 4). Also striking is that the marginal stability lines for the higher $m$ penetrate less and less into the region beyond the Rayleigh line, so that we have to consider the stratorotational instability as a "low- $m$ instability". Finally, we briefly discuss the applicability of these results to the stability problem of accretion disks, with their strong density stratification and rapid rotation.
\end{abstract}

Key words. accretion, accretion disks - hydrodynamics - turbulence

\section{Introduction}

The onset of instability in heterogeneous shear flows was studied by Thorpe (1968) who concluded that the density stratification stabilizes the flow. Further experimental and theoretical studies by Boubnov et al. (1995) and Caton et al. (2000) of flows between concentric rotating cylinders with a stable axial density stratification confirmed the stabilizing role of the density stratification and showed that i) the critical Reynolds number depends on the buoyancy frequency (or Brunt-Väisälä frequency) of the fluid; and ii) the stratification reduces the vertical extension of the Taylor vortices. The numerical results of Hua et al. (1997) have reproduced the experimental results.

Common to these studies is that the outer cylinder is at rest, and the flow is unstable according to the Rayleigh condition for inviscid flow, i.e.

$\frac{\mathrm{d} R^{4} \Omega^{2}}{\mathrm{~d} R}<0$,

with $\Omega$ as the angular velocity of the flow.

Recently, Molemaker et al. (2001) and Yavneh et al. (2001) found the much simpler relation

$\frac{\mathrm{d} \Omega^{2}}{\mathrm{~d} R}<0$

as the sufficient condition for (nonaxisymmetric) instability. The condition (2) is identical with the condition for magnetorotational instability of Taylor-Couette flow (Velikhov 1959).
These results have been derived by a linear stability analysis for inviscid flow. The numerical results of Yavneh et al. (2001) demonstrate the existence of the hydrodynamic instability also for finite viscosity.

According to Eq. (1) the rotation law $\Omega \propto R^{-2}$ plays an exceptional role. It separates the unstable-flow region from the stable-flow region and represents the "Rayleigh line". Withjack \& Chen (1974) experimentally found nonaxisymmetric disturbances in density-stratified Taylor-Couette flow beyond the Rayleigh line. With their wide-gap container $\left(R_{\text {in }}=0.2 \cdot R_{\text {out }}\right)$ they found the stability curve crossing the classical Rayleigh line. The resulting experimental instability line, however, is rather steep (see their Fig. 8) and never crosses the line $u_{\phi}=$ $R \Omega=$ const., which means that now the relation

$\frac{\mathrm{d} R^{2} \Omega^{2}}{\mathrm{~d} R}<0$

might serve as a new instability criterion. The results of our theory will confirm this idea.

For real, viscous flows there are illustrative results by Yavneh et al. (2001). In the present paper a more comprehensive study of such flows is given. The governing equations and the restrictions of the Boussinesq approximation are discussed in Sect. 2, while the numerical results are presented in Sect. 3. Summary and final discussion are given in Sect. 4.

The possible existence of an instability in Taylor-Couette flows with a stable radial rotation law and with a stable 


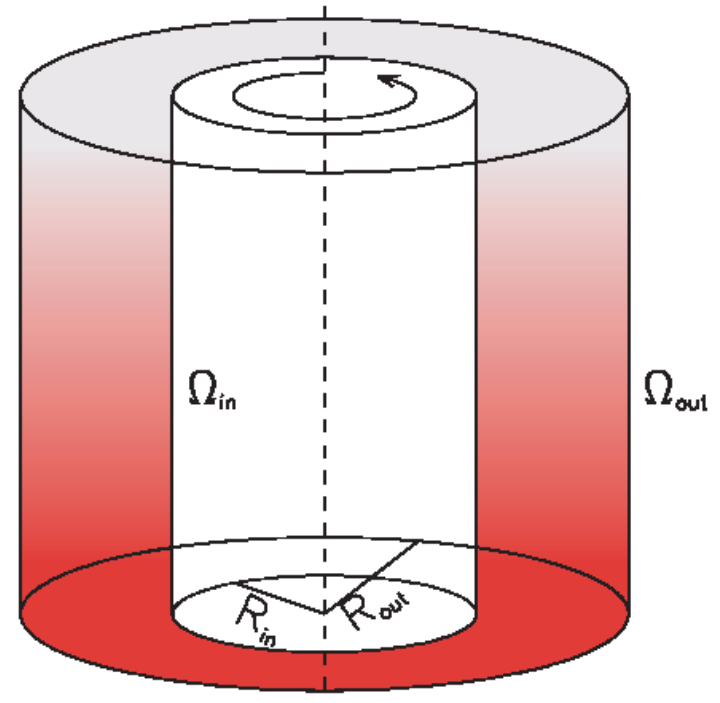

Fig. 1. The geometry of density-stratified Taylor-Couette experiments.

vertical stratification of the density is a surprise in the light of the Solberg-Høiland criterion (see Rüdiger et al. 2002). The necessary condition for stability reads

$\frac{\partial g_{R}}{\partial z}=\frac{\partial g_{z}}{\partial R}$

where $\boldsymbol{g}$ is the external force acceleration. Any conservative force is a particular solution of Eq. (4). Without external forces this relation is thus always fulfilled. If - as it is in accretion disks - the gravity balances the pressure and the centrifugal force, then Eq. (4) is automatically fulfilled. Note that according to the Poincare theorem for rotating media with potential force and $\Omega=\Omega(R)$, both the density and the pressure can be written as functions of the generalized potential so that Eq. (4) is always fulfilled. The magnetic field, however, is not conservative and can never fulfill the condition (4). This is the basic explanation for the existence of the magnetorotational instability (MRI) driven by (weak) magnetic fields.

Equation (4) has been derived by means of the short-wave approximation $m<R / \delta R$. For waves which are large in radial directions this condition might easily be fulfilled only for $m=0$. It is important therefore to go beyond the shortwave approximation in order to probe also the nonaxisymmetric modes. For Kepler flows (with uniform gravitational acceleration) this has recently been done by Dubrulle et al. (2005). With Boussinesq approximation and direct numerical simulations for viscous flow they find almost all stratified flows with negative $\mathrm{d} \Omega / \mathrm{d} R$ unstable against nonaxisymmetric disturbances. According to their results a critical Froude number (as defined by Eq. (24)) exists, below which the flow is stable ${ }^{1}$.

\footnotetext{
1 Whether the Boussinesq approximation can be used for too small Froude numbers seems still to be an open question.
}

\section{Equations and basic state}

In cylindrical coordinates $(R, \phi, z)$ the equations of an incompressible stratified fluid with uniform dynamic viscosity $\mu$ are

$$
\begin{aligned}
& \frac{\partial u_{R}}{\partial t}+(\boldsymbol{u} \nabla) u_{R}-\frac{u_{\phi}^{2}}{R}= \\
& -\frac{1}{\rho} \frac{\partial P}{\partial R}+v\left[\Delta u_{R}-\frac{2}{R^{2}} \frac{\partial u_{\phi}}{\partial \phi}-\frac{u_{R}}{R^{2}}\right], \\
& \frac{\partial u_{\phi}}{\partial t}+(\boldsymbol{u} \nabla) u_{\phi}+\frac{u_{\phi} u_{R}}{R}= \\
& -\frac{1}{\rho R} \frac{\partial P}{\partial \phi}+v\left[\Delta u_{\phi}+\frac{2}{R^{2}} \frac{\partial u_{R}}{\partial \phi}-\frac{u_{\phi}}{R^{2}}\right], \\
& \frac{\partial u_{z}}{\partial t}+(\boldsymbol{u} \nabla) u_{z}=-\frac{1}{\rho} \frac{\partial P}{\partial z}-g+v \Delta u_{z}, \\
& \frac{\partial u_{R}}{\partial R}+\frac{u_{R}}{R}+\frac{1}{R} \frac{\partial u_{\phi}}{\partial \phi}+\frac{\partial u_{z}}{\partial z}=0,
\end{aligned}
$$

where

$(\boldsymbol{u} \nabla) u_{R}=u_{R} \frac{\partial u_{R}}{\partial R}+\frac{u_{\phi}}{R} \frac{\partial u_{R}}{\partial \phi}+u_{z} \frac{\partial u_{R}}{\partial z}$

and

$\Delta u_{R}=\frac{\partial^{2} u_{R}}{\partial R^{2}}+\frac{1}{R} \frac{\partial u_{R}}{\partial R}+\frac{1}{R^{2}} \frac{\partial^{2} u_{R}}{\partial \phi^{2}}+\frac{\partial^{2} u_{R}}{\partial z^{2}}$.

$\rho$ is the density, $P$ the pressure, $g$ gravity, and $v=\mu / \rho$ the kinematic viscosity. The equation which describes the evolution of a density fluctuation moving in the nonuniform density field is

$\frac{\partial \rho}{\partial t}+(\boldsymbol{u} \nabla) \rho=0$

We have to formulate the basic state with prescribed velocity profile $\boldsymbol{u}=(0, R \Omega(R), 0)$ and given vertical density stratification $\rho=\rho(z)$. The system (5) then takes the form

$$
\begin{aligned}
& \frac{u_{\phi}^{2}}{R}=\frac{1}{\rho} \frac{\partial P}{\partial R}, \quad \frac{1}{\rho} \frac{\partial P}{\partial z}=-g, \\
& \frac{\partial^{2} u_{\phi}}{\partial R^{2}}+\frac{1}{R} \frac{\partial u_{\phi}}{\partial R}-\frac{u_{\phi}}{R^{2}}=0
\end{aligned}
$$

The last equation defines the angular velocity

$\Omega=a+\frac{b}{R^{2}}$,

where $a$ and $b$ are two constants related to the boundary values of the angular velocity, $\Omega_{\mathrm{in}}, \Omega_{\mathrm{out}}$, of the inner cylinder with radius $R_{\text {in }}$ and the outer cylinder with radius $R_{\text {out }}$. It follows

$a=\Omega_{\text {in }} \frac{\hat{\mu}-\hat{\eta}^{2}}{1-\hat{\eta}^{2}}, \quad b=\Omega_{\text {in }} R_{\text {in }}^{2} \frac{1-\hat{\mu}}{1-\hat{\eta}^{2}}$

with

$\hat{\mu}=\frac{\Omega_{\text {out }}}{\Omega_{\text {in }}}, \quad \hat{\eta}=\frac{R_{\text {in }}}{R_{\text {out }}}$.

The Rayleigh line is defined by $a=0$, i.e. $\hat{\mu}=\hat{\eta}^{2}$. Differentiating the first equation of the system (9) by $z$ and the second equation by $R$, subtracting each other and using the 
supposed profiles of density and angular velocity one finds the condition

$R \Omega^{2} \frac{\mathrm{d} \rho}{\mathrm{d} z}=0$.

According to this relation the density can depend only on the vertical coordinate $z$ in the absence of rotation and the angular velocity can only depend on radius in the absence of the vertical density stratification. The supposed profiles of the angular velocity, $\Omega=\Omega(R)$, and the density $\rho=\rho(z)$ are, therefore, not self-consistent. We have to admit more general profiles for the density $\rho=\rho(R, z)$ even though the initial stratification for the resting fluid is only vertical. In this case, the condition (13) takes the form

$R \Omega^{2} \frac{\partial \rho}{\partial z}+g \frac{\partial \rho}{\partial R}=0$.

The fluid transforms under the centrifugal force from the pure vertical stratification at the initial state to mixed (vertical and radial) stratification under the influence of the rotation so that the problem is strongly complicated.

For real experiments the initial (without rotation) vertical stratification is small $|\mathrm{d} \log \rho / \mathrm{d} \log z \ll 1|$ as is the ratio of centrifugal acceleration to the vertical gravitation acceleration

$\left|\frac{R^{2} \Omega}{g}\right| \ll 1$,

so that from Eq. (14) the radial stratification is also small. Let us therefore consider only a weak stratification

$\rho=\rho_{0}+\rho_{1}(R, z), \quad \rho_{1} \ll \rho_{0}$,

where $\rho_{0}$ is the uniform background density and Eq. (14) is fulfilled at zeroth-order. The perturbed state of the flow is described by $\boldsymbol{u}=\left(u_{R}, u_{\phi}+R \Omega(R), u_{z}\right)$ and

$P_{0}(R)+P_{1}(R, z)+P, \quad \rho_{0}+\rho_{1}(R, z)+\rho$,

where $\left|P_{1} / P_{0}\right| \ll 1$ and $\boldsymbol{u}, P$ and $\rho$ are the perturbations. Linearizing the system (5), (8) and selecting only the terms of the largest order we have

$$
\begin{aligned}
& \frac{\partial u_{R}}{\partial t}+\Omega \frac{\partial u_{R}}{\partial \phi}-2 \Omega u_{\phi}=-\frac{1}{\rho_{0}} \frac{\partial P}{\partial R}+\frac{\rho}{\rho_{0}^{2}} \frac{\partial P_{0}}{\partial R} \\
& +v_{0}\left[\Delta u_{R}-\frac{2}{R^{2}} \frac{\partial u_{\phi}}{\partial \phi}-\frac{u_{R}}{R^{2}}\right], \\
& \frac{\partial u_{\phi}}{\partial t}+\Omega \frac{\partial u_{\phi}}{\partial \phi}+\frac{1}{R} \frac{\partial}{\partial R}\left(R^{2} \Omega\right) u_{R}=-\frac{1}{\rho_{0} R} \frac{\partial P}{\partial \phi} \\
& +v_{0}\left[\Delta u_{\phi}+\frac{2}{R^{2}} \frac{\partial u_{R}}{\partial \phi}-\frac{u_{\phi}}{R^{2}}\right], \\
& \frac{\partial u_{z}}{\partial t}+\Omega \frac{\partial u_{z}}{\partial \phi}=-\frac{1}{\rho_{0}} \frac{\partial P}{\partial z}+\frac{\rho}{\rho_{0}^{2}} \frac{\partial P_{0}}{\partial z}+v_{0} \Delta u_{z}, \\
& \frac{\partial \rho}{\partial t}+\frac{\partial \rho_{1}}{\partial R} u_{R}+\Omega \frac{\partial \rho}{\partial \phi}+\frac{\partial \rho_{1}}{\partial z} u_{z}=0, \\
& \frac{\partial u_{R}}{\partial R}+\frac{u_{R}}{R}+\frac{1}{R} \frac{\partial u_{\phi}}{\partial \phi}+\frac{\partial u_{z}}{\partial z}=0
\end{aligned}
$$

with uniform $v_{0}=\mu / \rho_{0}$. The first-order terms are left in the mass conservation equation due to vanishing of the zerothorder terms.
Due to Eq. (15) we neglect $\partial P_{0} / \partial R$ in the first equation and $\partial \rho_{1} / \partial R$ in the fourth equation arising from radial stratification (they will be $\left|R^{2} \Omega / g\right|$ times smaller than terms arising from the vertical stratification) and the system takes the Boussinesq form

$$
\begin{aligned}
& \frac{\partial u_{R}}{\partial t}+\Omega \frac{\partial u_{R}}{\partial \phi}-2 \Omega u_{\phi}=-\frac{\partial}{\partial R}\left(\frac{P}{\rho_{0}}\right) \\
& +v_{0}\left[\Delta u_{R}-\frac{2}{R^{2}} \frac{\partial u_{\phi}}{\partial \phi}-\frac{u_{R}}{R^{2}}\right], \\
& \frac{\partial u_{\phi}}{\partial t}+\Omega \frac{\partial u_{\phi}}{\partial \phi}+\frac{1}{R} \frac{\partial R^{2} \Omega}{\partial R} u_{R}=-\frac{1}{R} \frac{\partial}{\partial \phi}\left(\frac{P}{\rho_{0}}\right) \\
& +v_{0}\left[\Delta u_{\phi}+\frac{2}{R^{2}} \frac{\partial u_{R}}{\partial \phi}-\frac{u_{\phi}}{R^{2}}\right], \\
& \frac{\partial u_{z}}{\partial t}+\Omega \frac{\partial u_{z}}{\partial \phi}=-\frac{\partial}{\partial z}\left(\frac{P}{\rho_{0}}\right)-g \frac{\rho}{\rho_{0}}+v_{0} \Delta u_{z}, \\
& \frac{\partial}{\partial t}\left(\frac{\rho}{\rho_{0}}\right)+\Omega \frac{\partial}{\partial \phi}\left(\frac{\rho}{\rho_{0}}\right)-\frac{N^{2}}{g} u_{z}=0, \\
& \frac{\partial u_{R}}{\partial R}+\frac{u_{R}}{R}+\frac{1}{R} \frac{\partial u_{\phi}}{\partial \phi}+\frac{\partial u_{z}}{\partial z}=0,
\end{aligned}
$$

where $N$ is the buoyancy frequency with

$$
N^{2}=-\frac{g}{\rho_{0}} \frac{\partial \rho_{1}}{\partial z}
$$

Suppose that the linear vertical density stratification $\partial \rho_{1} / \partial z=$ const. and thus $N$ is a constant, too. Then the coefficients of the system (19) depend only on the radial coordinate, and a normal mode expansion of the solution $F=F(R) \exp (\mathrm{i}(m \phi+k z+\omega t))$ can be used, where $F$ represents any of the disturbed quantities.

Let $D=R_{\text {out }}-R_{\text {in }}$ be the gap between the cylinders. We use $R_{0}=\left(R_{\mathrm{in}} D\right)^{1 / 2}$ as the unit of length, the velocity $\Omega_{\mathrm{in}} R_{0}$ as the unit of the perturbed velocity, $\Omega_{\text {in }}$ as the unit of $\omega, N$ and $\Omega$. Using the same symbols for normalized quantities and redefining $\rho$ as the dimensionless density $\rho g / \rho_{0} R_{0} \Omega_{\text {in }}^{2}$ we finally find

$$
\begin{aligned}
& \frac{\partial^{2} u_{R}}{\partial R^{2}}+\frac{1}{R} \frac{\partial u_{R}}{\partial R}-\frac{u_{R}}{R^{2}}-\left(k^{2}+\frac{m^{2}}{R^{2}}\right) u_{R}-2 \mathrm{i} \frac{m}{R} u_{\phi} \\
& \quad-\mathrm{i} \operatorname{Re}(\omega+m \Omega) u_{R}+2 \operatorname{Re} \Omega u_{\phi}-\operatorname{Re} \frac{\partial P}{\partial R}=0, \\
& \frac{\partial^{2} u_{\phi}}{\partial R^{2}}+\frac{1}{R} \frac{\partial u_{\phi}}{\partial R}-\frac{u_{\phi}}{R^{2}}-\left(k^{2}+\frac{m^{2}}{R^{2}}\right) u_{\phi}+2 \mathrm{i} \frac{m}{R} u_{R} \\
& -\mathrm{i} \operatorname{Re}(\omega+m \Omega) u_{\phi}-\mathrm{i} \operatorname{Re} \frac{m}{R} P-\frac{\operatorname{Re}}{R} \frac{\partial}{\partial R}\left(R^{2} \Omega\right)=0, \\
& \frac{\partial^{2} u_{z}}{\partial R^{2}}+\frac{1}{R} \frac{\partial u_{z}}{\partial R}-\left(k^{2}+\frac{m^{2}}{R^{2}}\right) u_{z} \\
& \quad-\mathrm{i} \operatorname{Re}(\omega+m \Omega) u_{z}-\mathrm{i} \operatorname{Re} k P-\operatorname{Re} \rho=0, \\
& \frac{\partial u_{R}}{\partial R}+\frac{u_{R}}{R}+\mathrm{i} \frac{m}{R} u_{\phi}+\mathrm{i} k u_{z}=0 \\
& \text { and } \\
& \mathrm{i}(\omega+m \Omega) \rho-N^{2} u_{z}=0
\end{aligned}
$$

with the Reynolds number

$\operatorname{Re}=\frac{\Omega_{\text {in }} R_{\text {in }} D}{v}$. 
The standard no-slip boundary conditions at the inner and outer cylinder (i.e. $u_{R}=u_{\phi}=u_{z}=0$ ) complete the classical eigenvalue problem. The same numerical method as in our previous papers about the Taylor-Couette problem (see e.g. Rüdiger et al. 2003) is used. Here a small negative imaginary part of $\omega$ to avoid problems with the corotation point $\omega=m \Omega$ for $m>0$ which is the critical point of the system (21) is adopted. The resulting critical Reynolds numbers are thus not for the marginally stable state but for the slightly unstable state. In order to be sure that the calculated unstable state can be realized in experiments we checked the existence of the transition from stable to unstable state for several sets of parameters.

The code has been tested by computing for $m=1$ the critical Reynolds number for the experiment run 2 of Withjack \& Chen (1974, their Table 1). The experimental value was 196.2 (in our normalizations) and our computed result is 200.6, which we accepted to be in sufficiently good agreement.

\section{Results}

The imaginary parts of $\omega, \mathfrak{J}(\omega)$, decrease with increasing Reynolds number (see Fig. 8 below). The Reynolds numbers above which $\mathfrak{J}(\omega)$ is smaller than some fixed value depend on the vertical wave number. They have a minimum at a certain wave number for fixed other parameters. This minimum value is called the critical Reynolds number.

In Fig. 2 we compare the calculated marginal stability line (i.e. $\mathfrak{J}(\omega)=0$ ) for axisymmetric disturbances with experimental values by Boubnov et al. (1995). The agreement is rather good except for small values of the Froude number

$\operatorname{Fr}=\frac{\Omega_{\text {in }}}{N}$.

Such disagreement may indicate the violation of the Boussinesq approximation. For Kepler disks one finds $\mathrm{Fr} \simeq 0.5$ (Dubrulle et al. 2005). The unstratified fluids possess infinite Froude number.

The dependence of the critical Reynolds numbers on $\hat{\mu}$ is given by Fig. 3 for a narrow gap and in Fig. 4 for a wide gap. The exact line of marginal stability is plotted only for $m=0$. The axisymmetric disturbances are unstable only for $\hat{\mu}<\hat{\eta}^{2}$ in accordance with the Rayleigh condition (1). For $m>0$ the slightly unstable lines with $\mathfrak{J}(\omega)=-10^{-3}$ are given.

Note that the nonaxisymmetric disturbances are unstable also beyond the Rayleigh line. However, the higher $m$ is, the less the corresponding instability line extends beyond from the Rayleigh line. Obviously, the "stratorotational instability" (SRI, Dubrulle et al. 2005) produces only low- $m$ modes. This is an indication that indeed it does not exist within the shortwave approximation (high- $m$ ) (Rüdiger et al. 2002).

For nonstratified Taylor-Couette flows the nonaxisymmetric modes are the most unstable disturbances only for counterrotating cylinders (Drazin \& Reid 1981). The nonaxisymmetric instability of the stratified Taylor-Couette flow beyond the Rayleigh line $\left(\hat{\mu}>\hat{\eta}^{2}\right)$ leads to the existence of some critical value, $\hat{\mu}_{\mathrm{c}}$, beyond which the nonaxisymmetric disturbances are the most unstable ones. Our results show that $\hat{\mu}_{\mathrm{c}} \sim 0.27$ and almost independent of the Froude number for $\hat{\eta}=0.78$ (small

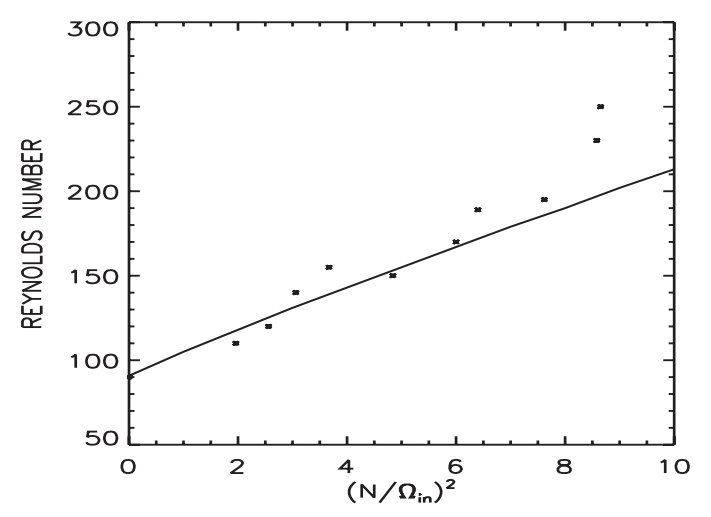

Fig. 2. The marginal stability line for axisymmetric disturbances $(m=$ 0 ) for $\hat{\eta}=0.78, \hat{\mu}=0$. The dots represent the experimental data of Boubnov et al. (1995).

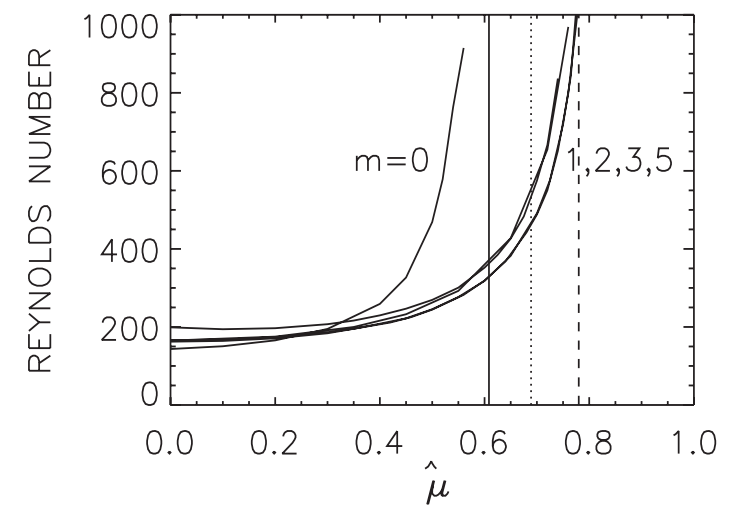

Fig. 3. The marginal stability line for $m=0$ and critical Reynolds numbers for $m>0$ for $\hat{\eta}=0.78$ and $\mathrm{Fr}=0.5$. The solid vertical line marks the Rayleigh line, the dashed vertical line marks $\hat{\mu}=\hat{\eta}$ and the dotted vertical line marks the "Kepler line" $\hat{\mu}=\hat{\eta}^{1.5}$.

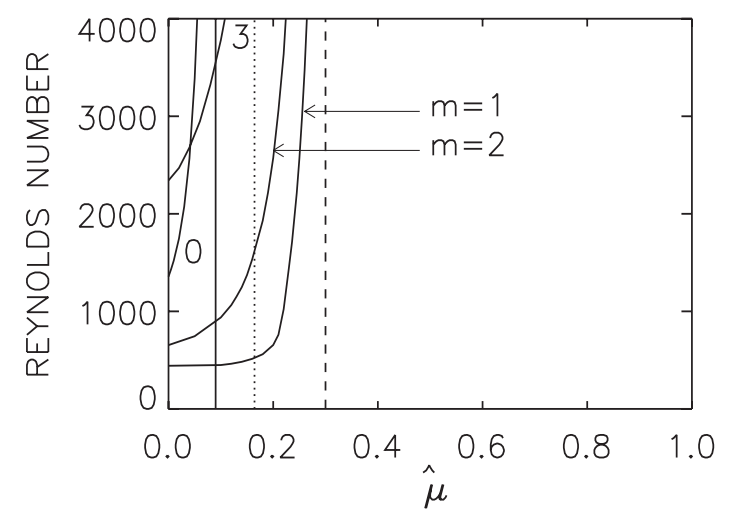

Fig. 4. The same as Fig. 3 but for a wide gap with $\hat{\eta}=0.3$.

gap) and $\hat{\mu}_{\mathrm{c}}<0$ for $\hat{\eta}=0.3$ (wide gap). It is even possible that for some $\hat{\eta}$ the nonaxisymmetric disturbances are the most unstable for all values of $\hat{\mu}$ corresponding to unstable flows.

Figures 3 and 4 contain another basic result. Approaching the line $\hat{\mu}=\hat{\eta}$ the instability lines become more and more steep. We did not find any solution for $\hat{\mu}>\hat{\eta}$. If this is true one has to change the condition (2) for the stratorotational instability to the condition (3). The condition (3) lies between the conditions (1) and (2); the allowed unstable rotation laws are flatter than the rotation laws of the Rayleigh instability but they are not 

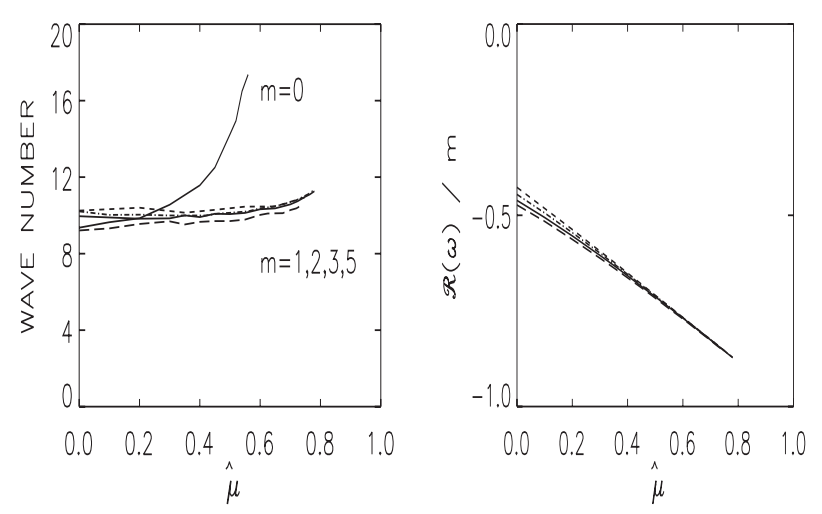

Fig. 5. The same as Fig. 3 but for vertical wave number (left $)$ and the pattern speed $\mathfrak{R}(\omega) / m$ for $m>0$ (right).
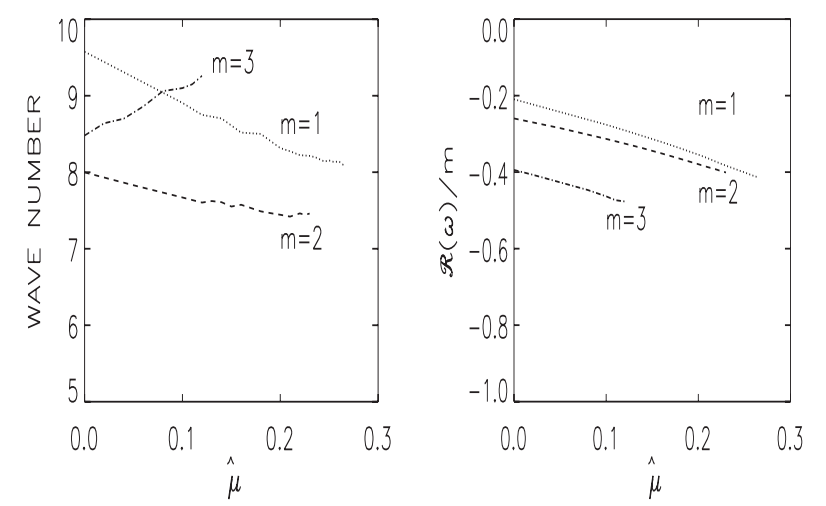

Fig. 6. The same as Fig. 5 but for wide gaps $(\hat{\eta}=0.3)$.

so flat as the flattest rotation laws which are unstable against MRI. This result seems to be of relevance for the discussion of the stability or instability of density-stratified Kepler disks.

For narrow gaps the critical Reynolds numbers of the nonaxisymmetric modes depend only slightly on the azimuthal wave number $m$. The same is true for the critical vertical wave number and the pattern speed $\mathfrak{R}(\omega) / m$ (Fig. 5). The vertical wave number depends only weakly on $\hat{\mu}$ and the values $\mathfrak{R}(\omega) / m$ vary linearly with $\hat{\mu}$. The situation is changed, however, for the wide gap $(\hat{\eta}=0.3)$. All parameters now depend strongly on both $m$ and $\hat{\mu}$ (Fig. 6). The trend for the vertical wave numbers form $m=3$ is opposite to those for $m=1$ and $m=2$.

The vertical wave numbers for both containers are of order 10 for $m=1$. With our normalization the vertical extent of the Taylor vortices is given by

$\frac{\delta z}{R_{\text {out }}}=\frac{\pi}{k} \sqrt{\hat{\eta}(1-\hat{\eta})}$

With the mentioned value of $k$ it is of order 0.1 for both the small-gap case and the wide-gap case. The cell becomes thus rather flat. For nonstratified TC-flows one finds $\delta z \simeq R_{\text {out }}-$ $R_{\text {in }}$, while the cells under the influence of an axial magnetic field become more and more prolate. The density stratification generally reduces the height of the Taylor vortices.

Unlike for the standard Taylor-Couette flow, $\mathfrak{R}(\omega)$ is not zero for density-stratified Taylor-Couette flow even for axisymmetric disturbances $(m=0)$. The onset of instability is thus
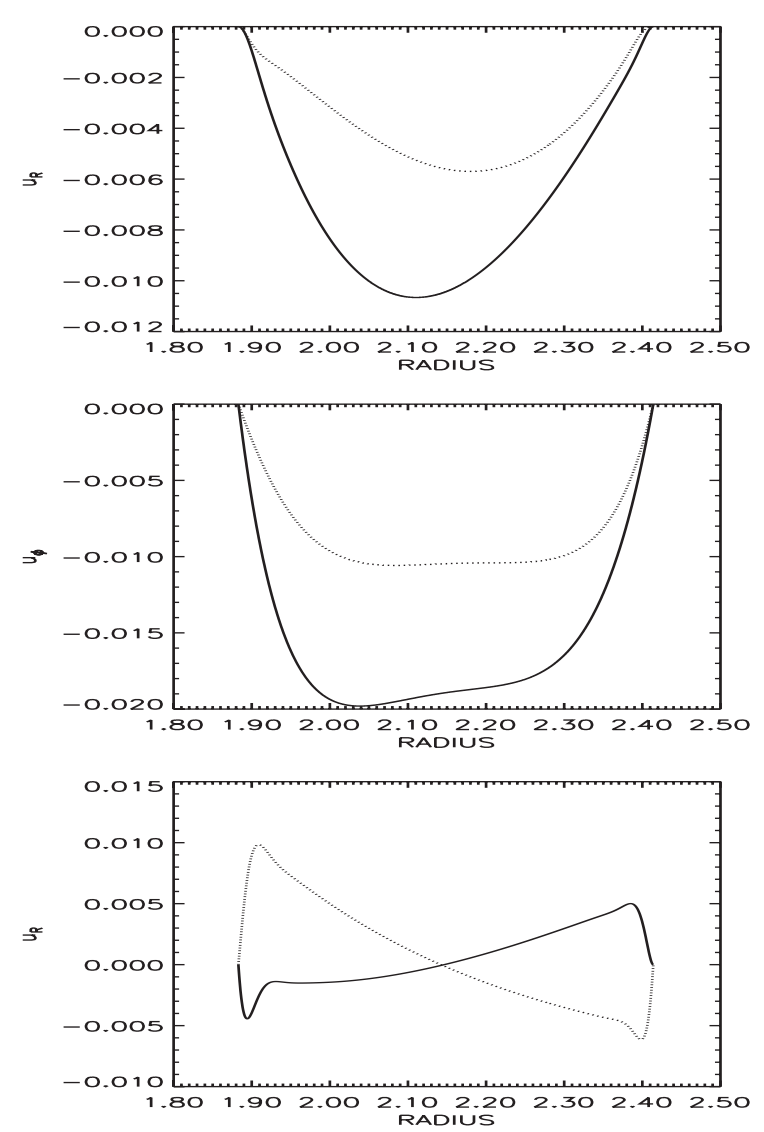

Fig. 7. The velocity eigenfunctions for $m=1, \hat{\eta}=0.78, \hat{\mu}=0.7$, $\mathrm{Fr}=0.5$ with Reynolds number, vertical wave number and $\mathfrak{R}(\omega)$. The dotted lines are the real part and solid lines are the imaginary part.

oscillatory. The question is whether a critical Froude number exists corresponding to the transition from stationary solutions to oscillating solutions. The answer is No. One cannot fulfill Eq. (22) for marginal stability $(\mathfrak{J}(\omega)=0)$ without a finite real part of $\omega$ for $N^{2} \neq 0$. The axially stratified Taylor-Couette flow bifurcates from the purely azimuthal flow through a direct Hopf bifurcation to a wavy regime. Depending on the value of $\hat{\mu}$ and $\hat{\eta}$ this new regime can be either oscillating and axisymmetric or nonaxisymmetric and azimuthally drifting (see Figs. 3 and 4). For both our containers the pattern speeds are negative for positive $m$. The drift of the spirals is thus always in the direction of the cylinder rotation.

Experiments have demonstrated the oscillating onset of the axisymmetric instability (e.g. Caton et al. 2000). It would be interesting to design experiments with either rotating outer cylinder or wider gap to probe the bifurcation from the overstable oscillating axisymmetric flow pattern to the spiral nonaxisymmetric flow pattern.

As an example, the velocity eigenfunctions are presented for the container with the narrow gap in Fig. 7, for $m=1$ and for $\hat{\mu}=0.7$, beyond the Rayleigh line. The functions are smooth, and do not suggest that the instability must be explained as a boundary effect.

The Reynolds number, the vertical wave number and the real part of $\omega$ for the transition from positive to negative imaginary part of $\omega$ are given in Fig. 8, i.e. for the transition 

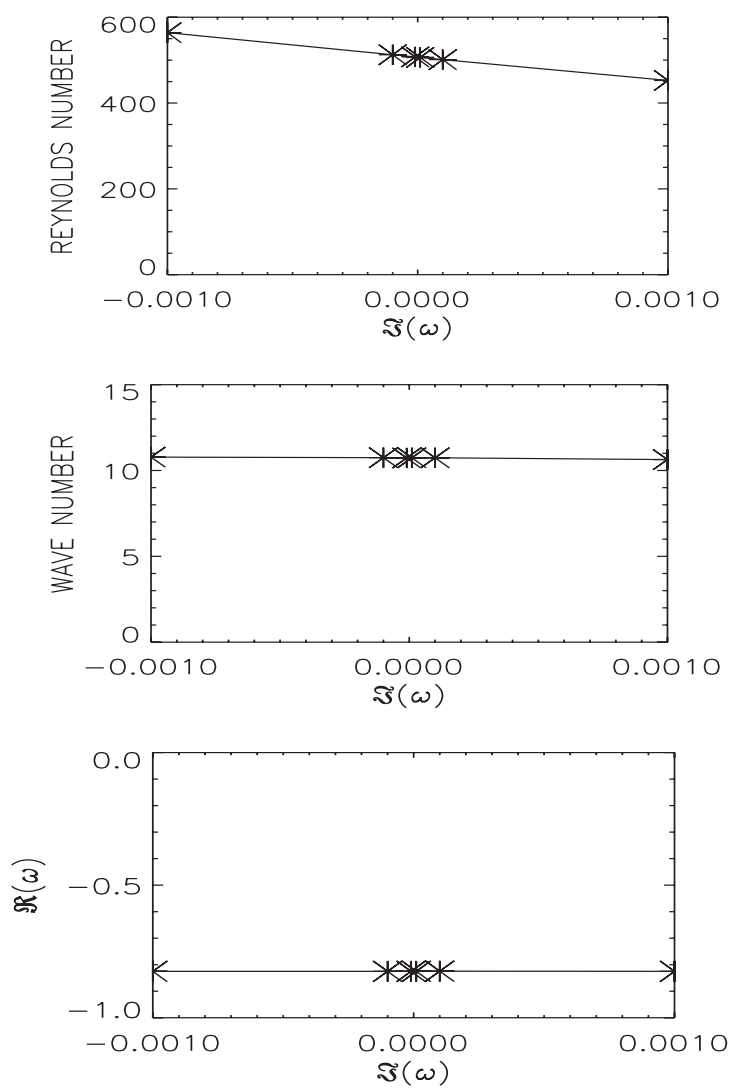

Fig. 8. The Reynolds number, vertical wave number and $\mathfrak{R}(\omega)$ in the transition from positive to negative $\mathfrak{J}(\omega)$ for $m=1, \hat{\eta}=0.78, \hat{\mu}=0.7$, $\mathrm{Fr}=0.5$.

from negative to positive growth rates. We find a continuous transition across the marginal stability line. It should thus be possible to realize the transition from stable to unstable flows in experiments. The vertical wave number and the real part of $\omega$ are hardly influenced by the transition but, not surprisingly, the Reynolds numbers has a remarkably clear trend.

\section{4. *Discussion}

It has been shown that density-stratified Taylor-Couette flow in the Boussinesq approximation yields nonaxisymmetric low $m$ instabilities that exist even beyond the Rayleigh line, i.e. for $\hat{\mu}>\hat{\eta}^{2}$. However, our results also show that the critical Reynolds numbers increase dramatically when approaching the line $\hat{\mu}=\hat{\eta}$, so that the instability condition appears to be

$\hat{\mu}<\hat{\eta}$

rather than $\hat{\mu}<1$ as suggested by Yavneh et al. (2001) and Molemaker et al. (2001). It is challenging to interpret the line $\hat{\mu}=\hat{\eta}$ with the (galactic) rotation profile $u_{\phi}=$ const in the same sense as to interpret the line $\hat{\mu}=\hat{\eta}^{2}$ with the rotation law for uniform specific angular momentum $R^{2} \Omega=$ const. Below we shall consider the line $\hat{\mu}=\hat{\eta}^{1.5}$ as concerning the Kepler flow ${ }^{2}$. Dubrulle et al. (2005) for their model of rotating plane Couette flow have found unstable solutions also beyond the line

2 Note, however, the general difference of the Kepler rotation law $\Omega \propto R^{-1.5}$ and the Taylor-Couette rotation law (10). $\hat{\mu}=\hat{\eta}$ but in these computations the rotation profile must also be steeper than $R^{-0.83}$ (their Fig. 6).

As our results are based on the Boussinesq approximation, two restrictions remain. The vertical density stratification should be weak enough and the rotation should be so slow enough that the centrifugal acceleration can be neglected. If one of these conditions is violated the Boussinesq approximation cannot be used and the situation becomes much more complicated. The disagreement between the calculated and the observed critical Reynolds numbers for small Fr (see Fig. 2) may indicate the violation of the Boussinesq approximation for stronger stratifications.

We have shown that for not too small negative $\mathrm{d} \Omega / \mathrm{d} R$ Taylor-Couette flows with vertical density stratification become unstable to nonaxisymmetric disturbances with low $m$ even if they are stable without density stratification. Kepler flows seem to be concerned by this phenomenon. In Figs. 3 and 4 the dotted lines represent the limit $\hat{\mu}=\hat{\eta}^{1.5}$, which may mimic the radial shear in Kepler disks. In both figures we find a critical Reynolds number of only about 500 for the lowest $(m=1)$ mode. This is a rather small number whose meaning, however, should not be overestimated. Approaching the line $\hat{\mu}=\hat{\eta}$ these values also become greater and greater. Even more important is the finding that in magnetohydrodynamic Taylor-Couette experiments the MRI needs magnetic Reynolds numbers of $\mathrm{O}(10)$. This leads to hydrodynamic Reynolds numbers exceeding $\mathrm{O}\left(10^{6}\right)$ only for experiments with liquid metals in terrestrial laboratories. For a hot plasma with magnetic Prandtl numbers of order 10 (Noguchi \& Pariev 2003) the necessary hydrodynamic Reynolds number is also only $\mathrm{O}(1)$ or even smaller!

The existence of the SRI might be important for astrophysical applications. As first suggested by Richard \& Zahn (1999) one should not forget (in particular for protoplanetary disks) to probe hydrodynamical instabilities as the source for the necessary turbulence in accretion disks. In partial confirmation of results of Dubrulle et al. (2005) we have here demonstrated that for Taylor-Couette flows under the presence of vertical density gradients linear hydrodynamic instabilities for low $m$ exist in contrast to the predictions of the Solberg-Høiland criterion. The properties of these modes are described above. Whether they are important for accretion disk physics is still an open question. Note that the density stratification in accretion disks vanishes in their equatorial region and that the unstable modes discussed above would more or less lead to a nonaxisymmetric structure of the disk rather than to turbulence. As the next step in this direction nonlinear simulations must reveal the amount of angular momentum which is transported by the SRI (Brandenburg \& Rüdiger 2005). Due to the rather smooth density profiles in their equatorial regions the numerical simulation for real global accretion disk models (see Barranco \& Marcus 2005) must follow.

Acknowledgements. D.S. is grateful for financial support from the AIP visitors program. His work was also partly supported by RFBR (grant 03-02-17522). G.R. thanks J.-P. Zahn for detailed discussions about the subject of this paper. Also the referee of this paper is cordially acknowledged for his interesting suggestions and remarks. 


\section{References}

Barranco, J. A., \& Marcus, P. 2005, ApJ, 623, 1157

Boubnov, B. M., Gledzer, E. B., \& Hopfinger, E. J. 1995, J. Fluid Mech., 292, 333

Brandenburg, A., \& Rüdiger, G. 2005, A\&A, in preparation

Caton, F., Janiaud, B., \& Hopfinger, E. J. 2000, J. Fluid Mech., 419, 93

Dubrulle, B., Marie, L., Normand, Ch., et al. 2005, A\&A, 429, 1

Drazin, P. G., \& Reid, W. H. 1981, Hydrodynamic stability (Cambridge: Cambridge Univ. Press)

Hua, B. L., Le Gentil, S., \& Orlandi, P. 1997, Phys. Fluids, 9, 365

Molemaker, M. J., McWilliams, J. C., \& Yavneh, I. 2001, Phys. Rev. Lett., 86, 5270
Noguchi, K., \& Pariev, V. 2003, Magnetorotational instability in a Couette flow of plasma, in Non-Neutral Plasma Physics V, AIP Conf. Proc., 692, 285

Richard, D., \& Zahn, J.-P. 1999, A\&A, 347, 734

Rüdiger, G., Arlt, R., \& Shalybkov, D. 2002, A\&A, 391, 781

Rüdiger, G., Schultz, M., \& Shalybkov, D. 2003, Phys. Rev. E, 67, 046312

Thorpe, S. A. 1968, J. Fluid Mech., 32, 693

Velikhov, E. P. 1959, Sov. Phys. JETP, 9, 995

Withjack, E. M., \& Chen, C. F. 1974, J. Fluid Mech., 66, 725

Yavneh, I., McWilliams, J. C., \& Molemaker, M. J. 2001, J. Fluid Mech., 448, 1 